> Les stratégies d'immunothérapie de l'infection par le VIH utilisant des cytokines ont pour objectifs d'interférer avec les mécanismes de dérégulation de l'homéostasie lymphocytaire $T$ induits par le virus, de potentialiser les effets des antiviraux sur la restauration immunitaire et de mobiliser les réservoirs du virus. Les interleukines 2 et 7 (IL-2 et IL-7) sont les cytokines les plus prometteuses. Un bénéfice clinique potentiel de ces stratégies est l'arrêt des traitements antiviraux, dont la toxicité à long terme est une préoccupation majeure. Par ailleurs, les effets d'une telle immuno-intervention sur la régulation de l'homéostasie lymphocytaire $T$ ou sur les fonctions immunologiques en présence du VIH nous renseignent également sur la physiopathologie de l'infection. <

Au cours des dix dernières années, les stratégies de combinaison de traitements antirétroviraux permettant de contenir de manière efficace la réplication virale ont changé l'évolution de l'infection par le VIH, en diminuant la mortalité et la morbidité des patients. Le bénéfice clinique est clairement lié à l'arrêt de la détérioration du système immunitaire secondaire à la réplication virale, ainsi qu'à la restauration quantative et qualitative des lymphocytes T CD4. Cependant, ces traitements antirétroviraux, qui ne permettent pas d'envisager une éradication du virus, ont transformé l'infection par le VIH en une infection chronique, qui nécessite un traitement continu à vie. Malgré la simplification des nouvelles combinaisons de traitement, celles-ci induisent un large éventail d'effets secondaires qui, pour l'instant, ne permettent pas de prédire leur possible utilisation chez un même patient sur plusieurs décennies. Des stratégies d'immunothérapie fondées sur l'administration de cytokines ont été proposées, et évaluées, dans l'infection par le VIH.

Article reçu le 26 mai 2006, accepté le 9 juin 2006.

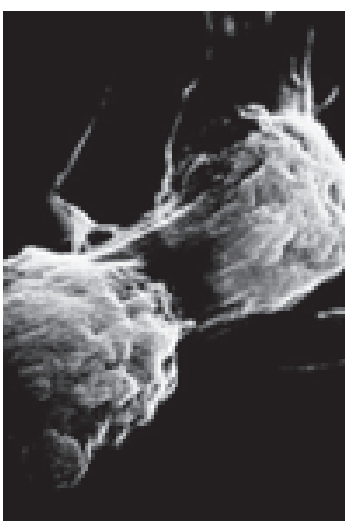

Service d'immunologie clinique, Hôpital Henri-Mondor,

51, avenue du Maréchal de Lattre de Tassigny, 94010 Créteil Cedex, France. yves.levy@hmn.aphp.fr

Traitement intermittent par l'interleukine-2

\section{Effet biologique}

L'administration de cures d'IL-2 de 5 jours, espacées de 6 à 8 semaines, peut entraîner une augmentation significative $d u$ taux de lymphocytes T CD4 chez les patients infectés par le VIH [1-7] (Figure 1). Cette augmentation est constamment supérieure à celle obtenue avec les traitements antirétroviraux. De plus, une telle augmentation a pu être démontrée chez des patients traités à différents stades de l'infection, et quel que soit le traitement antirétroviral associé à l'IL-2.

L'effet biologique de l'IL-2 est clairement dépendant de la dose administrée [8] : l'augmentation moyenne du taux des T CD4, par rapport au taux avant administration d'IL-2, est de 60,350 et 600 après 3 cures d'IL-2 à la posologie respectivement de 3 millions d'unités internationales (MUI), 9 MUI et 15 MUI par jour. Cependant, les effets secondaires sont également liés à la dose administrée, conduisant à une réduction des doses ou un arrêt de l'IL-2 chez environ $20 \%$ des patients ayant reçu la plus forte dose d'IL-2 [8]. Enfin, aucun effet délétère sur la réplication du virus n'a été noté in vivo, quels que soient la dose d'IL-2 administrée et le traitement antiviral associé.

Une observation clinique remarquable est le maintien à long terme (avec plus de 10 ans de recul) du taux des T CD4 induit par l'administration d'IL-2 $[9,10]$. Ainsi, on évalue à environ une cure, tous les 2-3 ans, le besoin d'IL-2 permettant de 
maintenir un taux de CD4 élevé après une phase d'induction d'environ 3 à 6 cures. Ces résultats démontrent que l'effet de I'IL-2 sur la restauration quantitative des lymphocytes T CD4 au cours de l'infection par le VIH est durable.

\section{Effets sur l'homéostasie lymphocytaire T}

Le traitement par l'IL-2 induit une augmentation significative du taux de lymphocytes T CD4 naïs et mémoires $[7,11]$. Au sein de la population mémoire, l'IL-2 augmente de manière prédominante la population mémoire centrale ( $\left.C D 45 R 0^{+} C D 27^{+}\right)$, alors que son effet est beaucoup plus faible, voire inexistant, sur les populations mémoires effectrices (CD45RO+ CD27-) [11]. Si l'effet quantitatif de l'IL-2 est sélectif de la population T CD4, une diminution de l'expression des marqueurs d'activation (comme le CD38) est notée sur la population T CD8.

Une caractéristique tout à fait étonnante du traitement par I'IL-2 est l'augmentation à long terme d'une population de lymphocytes TCD4 exprimant la chaîne $\alpha$ du récepteur de I'IL-2 (CD25) $[7,11]$; cette population peut représenter jusqu'à $60 \%$ des T CD4 après un an de traitement par l'IL-2 [7]. La majorité de ces cellules garde un phénotype de cellules T naïves ( $C D 45 R A^{+} C D 45 R 0^{-}$) ou intermédiaires $\left(C D 45 R A^{+} C D 45 R 0^{+}\right)$; elle n'est pas en cycle cellulaire, et n'exprime pas de marqueurs d'activation (HLA DR ou CD38) [12], ce qui indique que l'effet de I'IL-2 ne peut se résumer à une mise en cycle des T CD4. Au contraire, il semble que I'IL-2 diminue l'activation des lymphocytes T CD4, un paramètre impliqué dans la progression de la maladie et la diminution de la durée de vie des lymphocytes T CD4. Cette hypothèse a été récemment démontrée par l'administration, chez des patients infectés par le VIH, traités par IL-2 et recevant une perfusion de BrDU

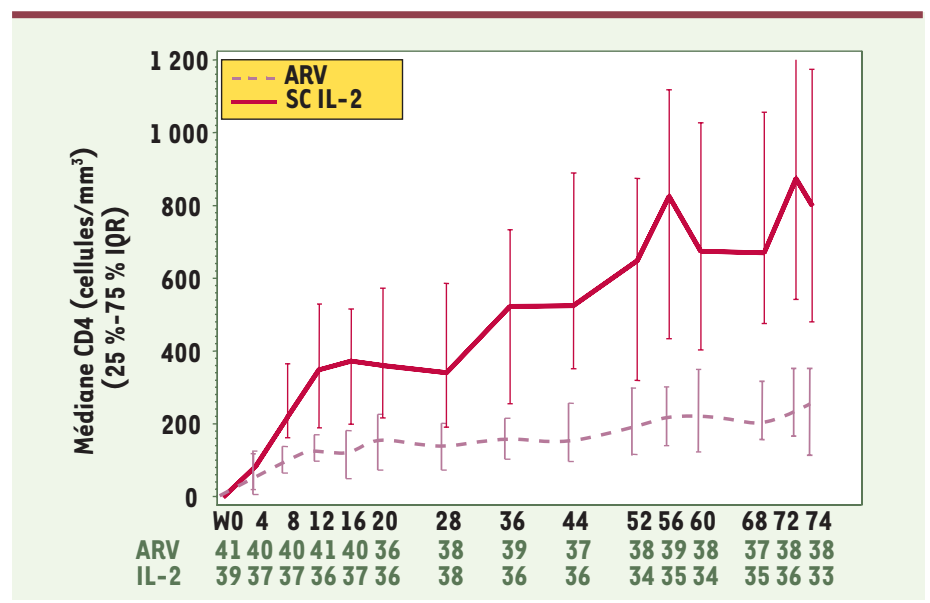

Figure 1. Profil d'augmentation des lymphocytes T CD4 sous traitement intermittent par l'IL-2 chez les patients infectés par le VIH. Les patients ont été traités par une combinaison d'antirétroviraux (ARV) administrés seuls (courbes hachurées) ou associés à des cures intermittentes d'IL-2 ( 5 MUI, deux fois par jour, pendant 5 jours, en sous-cutané), administrées toutes les 8 semaines. Après 18 mois de traitement, l'augmentation du taux des T CD 4 est de +250 cellules $/ \mu$ l et +850 cellules $/ \mu$, respectivement, chez les patients traités par ARV seuls ou en association avec l'IL-2 (résultats de l'essai ANRS 079) (d'après [7]). ou de glucose deutérium (permettant de suivre in vivo les lymphocytes T CD4 produits par l'administration de I'IL-2): les résultats montrent une augmentation significative de la survie des lymphocytes TCD4 sous IL-2 [14]. En effet, I'IL-2 induit une augmentation de la demi-vie des lymphocytes T CD4, qui est évaluée à 37 semaines contre 4 semaines chez des sujets non infectés, ou moins d'une semaine chez les patients infectés par le VIH et ne recevant pas d'IL-2. Ainsi, une stratégie d'immuno-intervention est capable d'interférer avec un des paramètres essentiels de la physiopathologie de I'infection par le VIH, celui de l'accélération de la mort cellulaire directement liée à l'infection virale ou à l'activation immunitaire.

Une retombée importante du traitement par l'IL-2 est donc l'amplification prédominante d'une population de $T C D 4^{+} C D 25^{+}$. Le phénotype de ces cellules est différent de celui des populations régulatrices T (T-reg) [14]. Bien qu'elles expriment le facteur de transcription FoxP3, elles ont un phénotype de T CD4 naïfs, prolifèrent in vitro en réponse aux antigènes de rappel et exercent une faible activité suppressive sur les réponses lymphocytaires [14]. Cependant, une faible proportion de cellules $C D 4^{+}$mémoires ( $C D 45 \mathrm{RO}^{+}$) exprimant fortement CD25 est également détectable chez les patients traités par IL-2 [15]. Quantitativement, cette population ne semble pas être modifiée par le traitement par IL-2. Ces cellules restent également capables de supprimer in vitro les réponses prolifératives des lymphocytes $T$ CD4 ${ }^{+}$CD25-, signant leur fonction de T-reg [15].

L'ensemble des observations réalisées indique que la population T CD4 amplifiée majoritairement in vivo par l'IL-2 a un phénotype particulier (CD45RO-CD25 $)$. Résulte-t-elle d'une population présente physiologiquement ou seulement chez les patients infectés par le VIH ? Sa contrepartie physiologique n'est pas connue. Cette population induite par l'IL-2 résiste à la mort cellulaire et reste peu activée malgré la réplication du VIH in vivo. Quels sont les mécanismes impliqués dans l'exceptionnelle longévité de cette population in vivo? La réponse à ces questions devrait, sans aucun doute, beaucoup nous en apprendre sur les mécanismes de l'homéostasie $T$ physiologiques et impliqués au cours de l'infection par le VIH. Le fait que le traitement par l'IL-2 affecte peu, sur les plans quantitatifs et fonctionnels, les cellules mémoires T régulatrices n'est pas non plus sans intérêt, même si le rôle précis de ces cellules sur l'activation cellulaire ou dans la physiopathologie de l'infection doit encore être éclairci [16, 17].

\section{Effets sur la fonction du système immunitaire}

Une question essentielle est de savoir si la fonction du système immunitaire est améliorée par le traitement par l'IL-2. 
Les tests permettant d'y répondre ne sont qu'indirects, et faiblement prédictifs d'un potentiel bénéfice clinique. De plus, le schéma d'administration de l'IL-2 reste vraisemblablement un facteur crucial pour ce type d'étude [18].

Plusieurs équipes ont analysé la réponse anticorps à des rappels de vaccination chez les patients infectés par le VIH et traités par l'IL-2 [7, 19]. La réponse IgG spécifique obtenue après rappel de vaccin antitétanique est meilleure lorsque I'IL-2 est associée au traitement antirétroviral [7]. Les réponses prolifératives TCD4 spécifiques d'antigènes de rappel sont également supérieures chez les patients traités par l'IL-2 [7]. L'utilisation de I'IL-2 comme adjuvant à une vaccination thérapeutique anti-VIH entraîne une augmentation de l'intensité des réponses T CD8 spécifiques du virus [20, 21]. L'ensemble de ces arguments est plutôt en faveur de la production de lymphocytes T CD4 mémoires fonctionnels sous IL-2.

Toutefois, le bénéfice clinique potentiel conféré par cette amélioration des fonctions du système immunitaire ne peut être évalué que par des essais cliniques de phase III, à grande échelle, évaluant la prévention des infections opportunistes et du sida chez les patients traités par IL-2. De tels essais (SILCAAT et ESPRIT) sont en cours, qui regroupent plus de 6500 patients (dont la moitié est traité par l'IL-2) dans plus de 10 pays $[22,23]$. Les résultats sont attendus pour 2008.

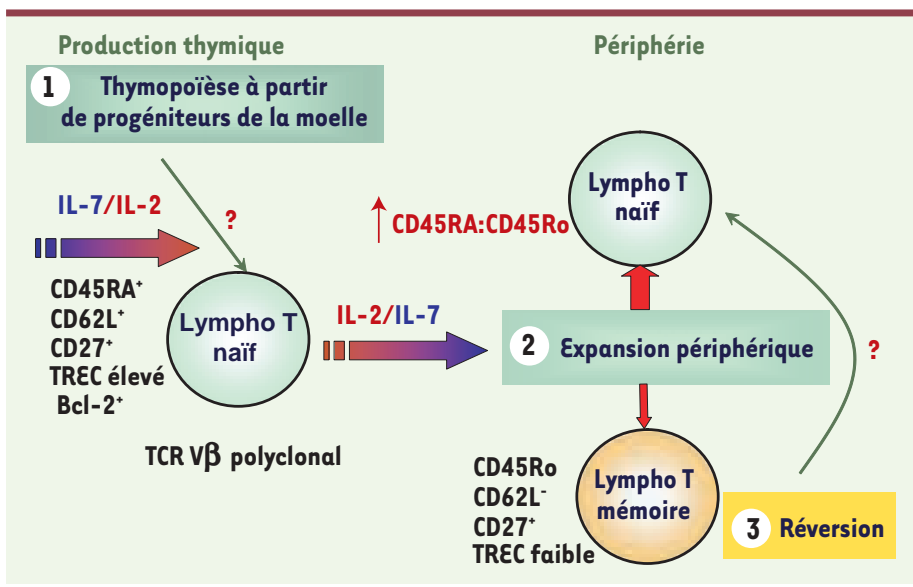

Figure 2. Effets de l'immunothérapie par IL-7 ou IL-2 associée aux antirétroviraux sur les mécanismes de restauration des lymphocytes T CD4. L'effet théorique de l'administration de I'IL-7 passerait par une augmentation de la thymopoïèse, une expansion périphérique des T CD4 et CD8 et, vraisemblablement, un effet sur la fonction (maturation) des lymphocytes T. Ces effets sont à l'étude dans des essais actuellement en cours, menés chez des patients infectés. L'IL-2 agit principalement sur le compartiment périphérique, et son effet est restreint à la population T CD4. II passe par une augmentation de la survie de la population amplifiée in vivo. L'IL-2 améliore les propriétés fonctionnelles des T CD4 et CD8 (réponses prolifératives testées in vitro et maturation des T CD8 in vivo). TREC: $T$ cell receptor excision circle, boucle d'ADN épisomique produit par le réarrangement du récepteur $T$ de l'antigène, et signature des émigrants thymiques récents.

\section{Intérêt potentiel du traitement par l'interleukine-7}

L'IL-7 joue un rôle fondamental dans la thymopoï̀se, I'homéostasie lymphocytaire T CD4 et CD8 périphérique et la survie cellulaire [24] (Figure 2). Dans l'infection par le $\mathrm{VIH}$, comme dans d'autres situations cliniques caractérisées par une lymphopénie, une corrélation inverse entre les concentrations plasmatiques d'IL-7 et l'importance de la lymphopénie a pu être mise en évidence $[25,26]$. Plusieurs études réalisées in vitro ont par ailleurs montré que l'IL-7 était capable d'induire la réplication du VIH à partir de cellules primaires infectées, ou après infection de monocytes ou de lymphocytes $T[27,28]$. Cependant, les modèles d'infection de macaques par le SIV (simian immunodeficiency virus) ont montré que l'administration d'IL-7 permettait d'augmenter le taux des lymphocytes T CD4 et CD8 sans augmentation de la réplication virale $[29,30]$. Ces données ont permis de mettre en place des essais de phase I d'évaluation de la tolérance de l'IL-7 chez les patients infectés par le VIH. Ces essais sont en cours en France et aux États-Unis, et les résultats attendus pour l'année 2007. L'intérêt potentiel de l'utilisation d'IL-7, en dehors de son effet sur l'homéostasie lymphocytaire $T$, est sa capacité à mobiliser in vitro des quasi-espèces virales à partir du réservoir viral lymphocytaire $T$ des patients infectés [31]. L'IL-7 pourrait mettre en jeu ce réservoir et son accessibilité à de nouvelles molécules antivirales ou des réponses immunitaires vis-à-vis de nouveaux épitopes ou variants viraux.

Si la démonstration d'un effet quantitatif de l'IL-7 sur les lymphocytes T des patients infectés par le VIH était faite, cette cytokine pourrait être utilisée en complément de thérapeutiques anvirales pour le maintien à long terme des T CD4, surtout chez les patients ayant une faible restauration immunitaire. L'association à des vaccinations thérapeutiques est également une des possibilités d'utilisation de l'IL-7, en raison de ses effets sur la maturation des cellules T CD8. Le bénéfice potentiel de cette cytokine, par rapport à l'IL-2, est sa longue demi-vie, qui laisse envisager la possibilité de l'administrer de façon espacée (2 à 3 fois/semaine, ou mensuellement).

\section{Conclusions}

Les stratégies d'immunothérapie de l'infection par le VIH utilisant des cytokines sont évidemment limitées par le faible nombre d'outils à notre disposition. Cependant, la longue expérience acquise avec l'IL-2 démontre qu'un traitement par cette cytokine est capable de restaurer quantitativement et qualitativement le taux des lymphocytes T CD4. L'action remarquable de cette cytokine sur la survie des lymphocytes T CD4 laisse également espérer l'action de certains traitements sur des facteurs clés de la physiopathogénie de l'infection par le VIH, à savoir l'activation du système immunitaire et la réduction de la demi-vie des lymphocytes T CD4 par une mort accélérée. Le bénéfice clinique à long terme de ces stratégies est encore toutefois à démontrer, même si la préservation du 
capital T CD4 n'est pas un enjeu trivial, en particulier chez les patients qui, pour des raisons d'effets secondaires ou de lassitude, seraient amener à interrompre un traitement antirétroviral. Bien que le taux des lymphocytes T CD4 et le contrôle de la réplication virale soient considérés comme des marqueurs fortement associés au pronostic de l'infection par le VIH, de même qu'à l'évaluation de l'efficacité des traitements antiviraux, la signification clinique d'une augmentation du taux de lymphocytes T CD4 sous traitement par l'IL-2 reste pour l'instant inconnue. Il est donc fondamental d'attendre la fin des essais de phase III qui permettront de répondre à ces questions. $\diamond$

\section{SUMMARY}

\section{Cytokine therapies in HIV infection}

The theoretical objectives of cytokine therapies in HIV infection are to impact $\mathrm{T}$ cell homeostasis and/or to improve immune functions or the mobilization of the HIV reservoir. Among cytokines, IL-2 and IL-7 are promising agents under clinical evaluation. Intermittent administration of IL-2 is by far the furthest studied strategy in HIV infection. This cytokine increases CD4 T lymphocytes in HIV-infected individuals. Recent clinical data showed that this effect is sustained over years. IL-2 therapy induces a peripheral expansion of T cells as a consequence of prolonged survival of T cells and decreased immune activation. These effects suggest that a cytokine therapy may interfere with critical factors of HIV disease. Recent data provide arguments that IL-2 therapy improves immune functions in HIV-infected patients. Whether these effects may be translated into clinical benefits is under evaluation in ongoing phase III studies. The potential interest of IL-7 in the treatment of HIV-infection is based on its crucial role on T cell homeostasis both in thymic output and peripheral T proliferation and survival. Although no data in human are still available, recent studies provide arguments to assess this cytokine in HIV infection. Phase I studies are ongoing or planned. $\diamond$

\section{RÉFÉRENCES}

1. Kovacs JA, Baseler M, Dewar RJ, et al. Increases in CD4 T lymphocytes with intermittent courses of interleukin-2 in patients with human immunodeficiency virus infection : a preliminary study. $N$ Engl J Med $1995 ; 332$ : 567-75.

2. Kovacs JA, Vogel S, Albert JM, et al. Sustained increases in CD4 counts with intermittent interleukin-2 therapy in HIV-infected patients with greater than $200 \mathrm{CD} 4$ cells $/ \mathrm{mm}^{3}$ : results of a randomized, controlled trial. $N$ Engl J Med 1996 ; 335 : 1350-6.

3. Davey RT, Chaitt DG, Piscitelli SC, et al. Subcutaneous administration of interleukin-2 in HIV1 infected persons. J Infect Dis $1997 ; 175: 781-9$.

4. Levy Y, Capitant C, Houhou S, et al. For the ANRS 048 study group. Comparison of subcutaneous and intravenous interleukin-2 in asymptomatic HIV-1 infection : a randomised controlled trial. Lancet 1999 ; 353 : $1923-9$.

5. Davey RT, Chaitt DJ, Albert JM, et al. A randomized trial of high-versus low-dose subcutaneous interleukin-2 outpatient therapy for early human immunodeficiency virus type 1 infection. J Infect Dis 1999; $179: 849-58$.

6. Davey RT, Murphy RL, Graziano FM, et al. Immunologic and virologic effects of subcutaneous interleukin-2 in combination with antiretroviral therapy: a randomized controlled trial. JAMA $2000 ; 284: 183-9$.

7. Levy Y, Durier C, Krzysiek R, et al. Effects of interleukin-2 therapy combined with highly active antiretroviral treatment on immune restoration in HIV-1 infection : a randomized controlled trial. AIDS $2003 ; 17: 343-51$.

8. Arduino RC, Nannini $\varepsilon C$, Rodriguez-Barradas M, et al. CD4 cell response to 3 doses of subcutaneous interleukin 2 : meta-analysis of 3 vanguard studies. Clin Infect Dis 2004 ; 39: 115-22.

9. Gougeon ML, Rouzioux C, Liberman I, et al. Immunological and virological effects of long term IL-2 therapy in HIV-1-infected patient. AIDS 2001 ; 15 : 1729-31.
10. Farel CE, Chaitt DG, Hahn BK, et al. Induction and maintenance therapy with intermittent interleukin-2 in HIV-1 infection. Blood 2004; $103: 3282-6$.

11. Sereti I, Martinez-Wilson H, Metcalf JA, et al. Long-term effect of intermittent interleukin-2 therapy in patients with HIV infection : characterization of a novel subset of $C D 4^{+} / C D 25^{+}$T cells. Blood 2002 ; $100: 2159-67$.

12. Sereti I, Anthony KB, Martinez-Wilson H, et al. IL-2-induced CD4 $4^{+} \mathrm{T}$ cell expansion in HIV-infected patients is associated with long term decreases in T-cell proliferation. Blood 2004 ; 104 : 775-80.

13. Kovacs JA, Lempicki RA, Sidorov IA, et al. Induction of prolonged survival of $\mathrm{CD}^{+}{ }^{+} \mathrm{T}$ lymphocytes by intermittent IL-2 therapy in HIV-infected patients. J Clin Invest $2005 ; 115: 2139-48$.

14. Sereti I, Imamichi $\mathrm{H}$, Natarajan $\mathrm{V}$, et al. In vivo expansion of $\mathrm{CD}^{+} \mathrm{CD} 45 \mathrm{R} \mathrm{O}^{-}$ CD25 ${ }^{+} \mathrm{T}$ cells expressing foxP3 in IL-2 treated HIV-infected patients. J Clin Invest $2005 ; 115$ : 1839-47.

15. Weiss L, Donkova-Petrini V, Aouba A, Levy Y. CD $4^{+} C D 25^{+}$regulatory T cells are detectable in peripheral blood of HIV-infected patients treated with IL-2. Rio de Janeiro: $3^{\text {rd }}$ IAS Conference, 2005: We.Pe.16.5.B06 (abstract).

16. Weiss, L, Donkova-Petrini, V, Caccavelli, L, et al. Human immunodeficiency virus-driven expansion of $C D 4^{+} C D 25^{+}$regulatory $T$ cells which suppress HIV-specific CD4 T-cell responses in HIV-infected patients. Blood $2004 ; 101: 3249-56$.

17. Kinter $A$, Hennessey M, Bell A, et al. $C D 25^{+} C D 4^{+}$regulatory T cells from the peripheral blood of asymptomatic HIV-infected individuals regulate $\mathrm{CD} 4^{+}$and $\mathrm{CD} 8^{+}$HIV-specific T cell immune responses in vitro and are associated with favourable clinical markers of disease status. J Exp Med $2004 ; 200: 331-43$.

18. Blattman JN, Grayson JM, Wherry EJ, et al. Therapeutic use of IL-2 to enhance antiviral T-cell responses in vivo. Nat Med $2003 ; 9: 540-7$.

19. Valdez H, Mitsuyasu R, Landay A, et al. Interleukin-2 induces increases in $C D 4^{+}$lymphocyte numbers but does not enhance responses to immunization: results of A5046s. J Infect Dis 2003; 187 : 320-5.

20. Levy Y, Gahery-Segard H, Durier C, et al. Immunological and virological efficacy of a therapeutic immunization combined with interleukin-2 in chronically HIV-1 infected patient. AIDS 2005 ; 19 : 279-86.

21. Barouch DH, Santra S, Steenbeke TD, et al. Augmentation and suppression of immune responses to an HIV-1 DNA vaccine by plasmid cytokine/Ig administration. J Immunol 1998; 161 : 1875-82.

22. ESPRIT IL-2 trial (online) http : //www.espritstudy.org.

23. SILCAAT study design (online) http: //www.silcaatstudy.org.

24. Fry TJ, Mackall C. Interleukin-7 : from bench to clinic. Blood 2002 ; 99 : 3892-904.

25. Bolotin $\varepsilon$, Annett G, Parkman R, Weinberg K. Serum levels of IL-7 in bone marrow transplant recipients : relationship to clinical characteristics and lymphocyte count. Bone Marrow Transplant 1999; 8 : 783-8.

26. Napolitano LA, Grant RM, Deeks SG, et al. Increased production of IL-7 accompanies HIV-1-mediated T-cell depletion. Implications for T cell homeostasis. Nat Med $2001 ; 7: 73-9$.

27. Smithgall MD, Wong JG, Critchett KE, Haffar OK. IL-7 up-regulates HIV-1 replication in naturally infected peripheral blood mononuclear cells. J Immunol 1996; $156: 2324-30$.

28. Steffens CM, Managlia EZ, Landay A, Al-Harthi L. Interleukin-7 treated naïve $T$ cells can be productively infected by $T$-cell-adapted and primary isolates of human immunodeficiency virus 1 . Blood $2002 ; 99: 3310-8$.

29. Fry TJ, Moniuszko M, Creekmore S, et al. IL-7 therapy dramatically alters peripheral T-cell homeostasis in normal and SIV-infected nonhuman primates. Blood 2003; 101 : 2294-9.

30. Nugeyre MT, Monceaux V, Beq S, et al. IL-7 stimulates T cell renewal without increasing viral replication in simian immunodeficiency virusinfected macaques. J Immunol 2003 ; 171 : 4447-53.

31. Wang FX, Xu Y, Sullivan J, et al. IL-7 is a potent and proviral strainspecific inducer of latent HIV-l cellular reservoirs of infected individuals on virally suppressive HAART. J Clin Invest 2005 ; 115 : 128-37.
TIRÉS À PART

Y. Lévy 